\title{
Computed tomography of complicated Meckel's diverticulum in adults: a pictorial review
}

\author{
Alexandra Platon • Pascal Gervaz • \\ Christoph D. Becker • Philippe Morel • \\ Pierre-Alexandre Poletti
}

Received: 19 November 2009 / Accepted: 2 March 2010/Published online: 14 April 2010

(C) European Society of Radiology 2010

\begin{abstract}
Objective To show various CT aspects of complicated Meckel's diverticulum in adult patients to facilitate the preoperative diagnosis of this rare pathology in emergency settings.

Methods A computer search of medical records over a 15 year period identified 23 adult patients who underwent surgery for acute abdomen generated by a complicated Meckel's diverticulum. CT images available for review were analyzed, and some specific patterns leading to the diagnosis of complicated Meckel's diverticulum are presented in this review.

Results Complications were related to inflammation (14 patients), bleeding (5 patients), intestinal obstruction (3 patients), and penetrating foreign body (1 patient). The presence of a Meckel's diverticulum was usually suggested at CT scan by an abnormal outpouching, blind-ending digestive structure connected to the terminal ileum by a neck of variable caliber. Depending on the type of complications, the diverticulum was surrounded by mesenteric inflammatory changes, or presented as a localized fluid or air-fluid collection contiguous with the terminal ileum. The diverticulum was also the source of active bleeding or acted as the lead point to intestinal obstruction or intussusception.

Conclusion CT findings of complicated Meckel's diverticulum are polymorphic and should be considered in the evaluation of adult patients with acute abdomen.
\end{abstract}

\footnotetext{
A. Platon $(\varangle) \cdot$ C. D. Becker $\cdot$ P.-A. Poletti

Department of Radiology, University Hospital of Geneva,

4, rue Gabrielle-Perret-Gentil,

1211 Geneva, Switzerland

e-mail: alexandra.platon@hcuge.ch

P. Gervaz $\cdot$ P. Morel

Department of Surgery, University Hospital of Geneva,

Geneva, Switzerland
}

Keywords Meckel's diverticulum · Computed tomography $\cdot$ Acute abdomen $\cdot$ Complications $\cdot$ Bowel

\section{Introduction}

Meckel's diverticulum is a congenital anomaly of the gastrointestinal system due to incomplete obliteration of the omphalomesenteric duct. Meckel's diverticulum is found with an incidence of $1-2 \%$ in the general population, and most of the diverticula remain asymptomatic [1]. Complications result most commonly from bleeding, inflammation, and obstruction [1,2]. A study performed over a 42 year period in a general population showed a lifetime risk of complications of $6.4 \%$ [3]; symptomatic Meckel's diverticulum has been reported in $16 \%$ of patients with surgically proven diverticula [4]. According to this latter study, younger ( $<4$ years) patients tend to present with obstructions, whereas adult patients tend to present with hemorrhage. Complicated Meckel's diverticulum usually manifests during the first years of life, and it is considered to be a rare pathology in adult life.

The purpose of our review is to show various CT aspects of complicated Meckel's diverticulum in adult patients encountered over a 15 year period in our emergency department. We aim to facilitate the diagnosis of complicated Meckel's diverticulum prior to surgery in emergency conditions.

\section{Anatomy and embryology of the Meckel's diverticulum}

The presence of the Meckel's diverticulum is explained by the intrauterine evolution of the bowel. The initial embryological communication between the midgut and the yolk sac is made through the omphalomesenteric (vitelline) duct. During normal developmental evolution, this duct is 
obliterated and disappears; this process usually takes place during the 8th to 9th week of embryonic life. Different forms of congenital anomalies will result as a consequence of failure of closure and/or resorption of this embryological communication. The pattern of these anomalies depends on the degree of persistent ductal patency linking the ileum to the umbilicus; they may appear as fibrous cord, as ileoumbilical fistula, or as omphalomesenteric cysts $[2,5]$.

However, the most frequent of all closure anomalies of the omphalomesenteric duct is Meckel's diverticulum, which received its name in 1809 , in honor of the German anatomist Johann Friedrich Meckel. Meckel's diverticulum development is related to the persistent patency of the intestinal attachment of the omphalomesenteric duct, while its umbilical end obliterates. The result will be a true diverticulum, formed by all the layers of the intestinal wall; the diverticulum takes its origin from the antimesenteric border of the small bowel, within $40-100 \mathrm{~cm}$ of the ileocecal valve $[2,5]$. The arterial supply comes from the vitelline artery, which is a branch of the superior mesenteric artery. The diverticulum is normally lined by intestinal mucosa, but a frequent histological finding is the presence of heterotopic gastric or pancreatic mucosa [2].

\section{Clinical presentation}

The majority of Meckel's diverticulum cases remain clinically silent during the entire lifetime, and their presence may be discovered incidentally during surgery, autopsy, or when performing upper gastrointestinal studies $[2,5]$.

Bleeding is a frequent complication of Meckel's diverticulum. It is reported to be more frequent in children and young adults and is usually associated with ulcerations produced by the acid secretion from the heterotopic gastric mucosa inside the diverticulum. The bleeding may present as acute massive hemorrhage or as anemia from chronic bleeding; stools can be melenic or have a currant jelly-like aspect $[2,5]$.

Another frequent complication associated with Meckel's diverticulum is intestinal obstruction. Clinical symptoms are nonspecific; patients may have abdominal pain and distension, vomiting and constipation. Intestinal obstruction occurs due to a volvulus or an internal hernia around a diverticulum associated with a fibrotic band attached to the abdominal wall, intussusception, or incarceration of the diverticulum in an inguinal (Littré) hernia [5].

Inflammation of the Meckel's diverticulum leading to diverticulitis may be due to peptic ulceration from acid secretion related to heterotopic gastric mucosa or to obstruction of the diverticular neck by enteroliths or foreign bodies, with consecutive stasis and infection [2].

Diverticulitis is a complication that is more commonly reported in adult patients [1]. Clinical symptoms of
Meckel's diverticulitis are frequently identical to the symptoms of acute appendicitis, with patients presenting with right lower quadrant pain, fever, and vomiting. Meckel's diverticulitis may be complicated by perforation and peritonitis or may lead to inflammatory adhesion and intestinal obstruction.

\section{Imaging of complicated Meckel's diverticulum}

Meckel's diverticulum-related complications occur so infrequently that most articles have reported small series or isolated cases [6, 7]; in addition, many patients with Meckel's diverticulitis have nonspecific abdominal complaints, often making preoperative correct diagnosis difficult [8]. Traditionally, clinicians, when confronted with a patient with complicated Meckel's diverticulum, relied on conventional gastrointestinal contrast studies, angiography, or scintigraphy $[5,8]$. These methods have been progressively replaced by computed tomography scan (CT), which has become routinely used as the first-line imaging tool in the diagnostic work-up of the acute abdomen $[6,9,10]$.

Radionuclide studies have some specific indications in diagnosing bleeding of a Meckel's origin. Scintigraphy using Tc-99 m pertechnetate is targeted to the heterotopic gastric mucosa inside the Meckel's diverticulum and is used mainly on pediatric patients [11]. The sensitivity and the specificity of this method decrease in adult patients, due to the lower prevalence of gastric mucosa in symptomatic Meckel's diverticulum in adult patients [2]. In addition, this method is dedicated to the etiological diagnosis of occult, intermittent gastrointestinal bleeding and, therefore, it is not suitable for patients in unstable condition due to hemorrhage.

Scintigraphy with Tc-99 m-labelled red blood cells is the most sensitive imaging method to detect active gastrointestinal hemorrhage with bleeding rates as low as $0.1 \mathrm{ml} / \mathrm{min}$ [12]. Despite this enormous advantage and its ability to offer prolonged examination time, it remains less used in emergency settings. The drawbacks of the method include its incapacity to clearly show the anatomic origin of the bleeding, the inherent long imaging time, and the sometimes-limited hospital availability in emergency conditions [13].

Angiographic diagnosis of the Meckel's diverticulum lies in the identification of a persistent vitelline artery. This artery arises from the distal ileal branches and consists of a long vessel with few branches extending over the vasa recta of the adjacent ileal arteries; small tortuous branches at the distal part of this artery along with mucosal blush of the Meckel's diverticulum can also be seen $[14,15]$. In a small number of cases, patients with surgically proven Meckel's diverticulum may have normal angiograms $[14,16]$. 
During arteriography, contrast material extravasation into the bowel lumen is the most obvious sign of active gastrointestinal bleeding; this usually occurs when the bleeding rate is higher than $0.5 \mathrm{ml} / \mathrm{min}$. Accumulation of contrast in a space connected with the bowel lumen suggests a bleeding diverticulum [12]. Embolization of the bleeding vessel may control life-threatening hemorrhage from a Meckel's diverticulum in poor surgical candidates. While embolization is not the final method in treating Meckel's diverticulum, it may still act as a bridge toward surgery under more favorable hemodynamic situations [17].

\section{CT examination}

For a long time, CT was considered of little interest in diagnosing Meckel's diverticulum because of its suspected incapacity to differentiate the diverticulum from a loop of small bowel [5]. However, our observations from the current series, along with published reports concerning both adult and child patients $[6,10]$ reveal new opportunities for $\mathrm{CT}$ in diagnosing this pathology.

The CT pattern associated with the presence of Meckel's diverticulum is that of an outpouching blind-ended digestive structure arising from the antimesenteric side of the terminal ileum; this structure is found in the right lower quadrant or lower abdomen and is connected to the small bowel by a neck of varying caliber $[6,18]$. The diverticulum is considered complicated if it is the site of bowel obstruction or if it presents surrounding features of infiltration or inflammation, signs of perforation, adjacent fluid collection, or active bleeding.

While there is no clinical presentation that is specific to a complicated Meckel's diverticulum, the CT examination protocol is adapted to the patients' clinical presentation and follows some general rules in assessing the origin of acute abdomen [9].

First, the CT is usually performed with intravenous contrast medium injection. Intravenous injection of contrast medium has several advantages: among other things, it allows the assessment of the normal or pathological enhancement of the digestive and extra-digestive structures, the presence and extent of inflammatory infiltration, and the evaluation of the intraabdominal vessels. The volume of injected contrast medium is adapted to the patient's weight and ranges from 100 to $150 \mathrm{ml}$ of nonionic contrast medium.

If a patient is referred for diffuse abdominal pain, to assess intraabdominal collections, or for signs of perforation, administration of water-soluble oral contrast material is helpful in delineating the digestive structures and, therefore, distinguishing between bowel loops and extradigestive collections. While looking for a Meckel's diverticulum, it is of particular interest to assess the different structures in the right lower quadrant, with a special mention for the caecum, appendix, and the terminal ileum $[6,9]$.

However, the use of oral contrast has to be adapted to the clinical conditions that mandated the CT examination. Administration of water-soluble oral contrast material before scanning is possible only when the clinical condition of the patient allows waiting at least $1 \mathrm{~h}$ before the CT examination. Oral contrast material is completely prohibited in a patient with active bleeding because it may obscure the site of hemorrhage [9].

A CT angiography protocol is highly useful when evaluating patients with acute gastrointestinal bleeding. CT angiography involves a sequential acquisition of images of the entire abdomen. First, unenhanced scanning of the abdomen is performed to identify the pre-existing spontaneous hyperattenuating material in the bowel lumen, which could be mistaken for hemorrhage. Afterwards, scanning is performed sequentially for the arterial and portal vein phases and also at a delayed time after intravenous contrast injection, in order to show both the active contrast extravasation and to identify the underlying source of bleeding [13, 19].

Generally, CT angiography is considered diagnostic for active bleeding when there is high-attenuating contrast material extravasating into the bowel lumen that was not reported at unenhanced $\mathrm{CT}$ acquisition, performed prior to intravenous contrast injection [13]. Moreover, contrast extravasation and accumulation in a blind-ending digestive structure attached to the terminal ileum may be highly evocative for the presence of a Meckel's diverticulum [16]. Negative CT results are related to the intermittent nature of the bleeding or to the low extravasation rate, not seen on different acquisition sequences.

\section{Image review}

Our article reviews the CT scan findings of complicated Meckel's diverticulum in adult patients encountered over a 15 year period in our emergency department, which provides medical assistance for over 50,000 emergency patients per year. The computer search of medical records identified 23 adult patients (more than 18 years old) who underwent surgery for acute abdomen generated by a complicated Meckel's diverticulum. Our study group was composed of 12 men and 11 women, with a mean age of 42.6 years (range 20-86 years). The complications related to the diverticulum that were demonstrated at surgery were inflammation in 14 patients, bleeding in 5 patients, intestinal obstruction in 3 patients, and penetrating foreign body in 1 patient. There were no calcified enteroliths in the complicated Meckel's diverticulum, substantiating the assertion that this is a rare finding $[6,10]$.

The CT images available for review have been analyzed, and some specific patterns that could lead to the diagnosis 
of complicated Meckel's diverticulum are hereby presented in our review.

\section{Inflammation}

Diverticulitis was the most common presentation of a complicated Meckel's diverticulum in our patients.

According to our observations, the most straightforwardly recognizable CT scan appearance of Meckel's diverticulitis was that of a tubular or rounded blind-ending digestive structure, attached to a distal ileal loop and surrounded by mesenteric fat infiltration (Fig. 1). The diverticulum may be fluid, air-fluid, or contrast filled and is situated either in the right lower quadrant or in the lower abdomen, with normal ileum distal and proximal to its attachment point.

The identification of the attachment point of the diverticulum to the distal ileum allowed us to recognize with an important degree of certainty the presence of Meckel's diverticulum on CT scan. For this purpose, the presence of oral contrast inside the diverticulum provides a better characterization of its ileal attachment, by allowing a good visualization of the pattern of the mucosal folds on the junction region. The continuation of the mucosal folds from the ileum into the diverticulum may form a "triradiate" mucosal pattern, if ileal loops are collapsed, or may look like a "triangular plateau," if ileal loops are distended by the oral contrast. This appearance has already been described on gastrointestinal contrast examination of the small bowel $[2$, 5] and is considered pathognomonic for the diagnosis of Meckel's diverticulum; herein, for the first time, we report its aspect on a CT scan examination (Fig. 2).

More severe instances of Meckel's diverticulitis may appear as an abscess (Fig. 3) or as an inflammatory mass (Fig. 4) in contact with the terminal ileum [20, 21]. An air-

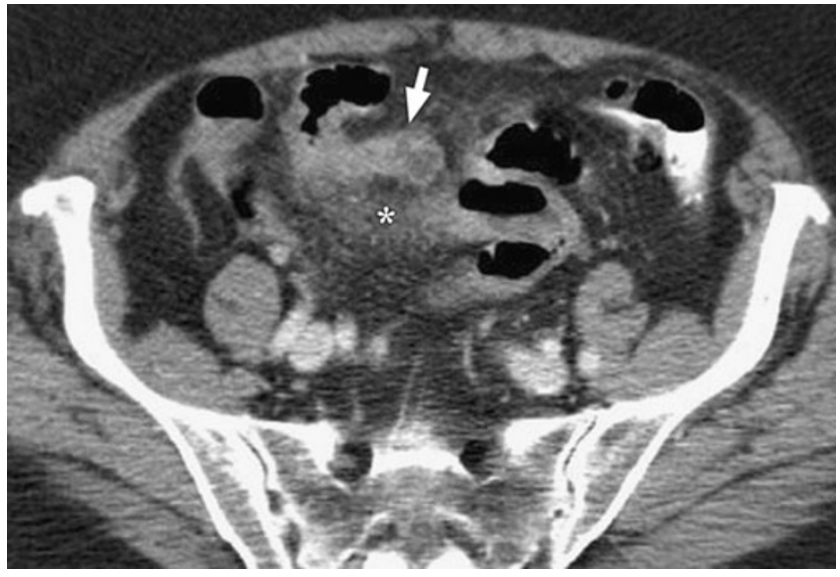

Fig. 1 A 76-year-old patient with surgically proven Meckel's diverticulitis. Intravenous contrast-enhanced CT shows the Meckel's diverticulum as a fluid-filled, blind-ending digestive structure (arrow), arising from a distal ileal loop, along with peridiverticular mesenteric fat stranding (asterisk), suggestive of inflammation.

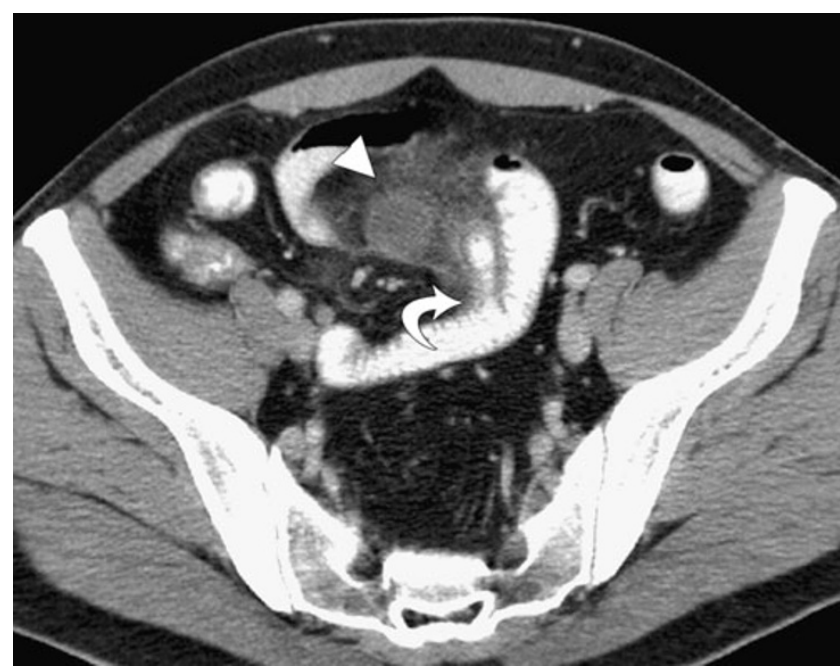

Fig. 2 A 37-year-old man with inflamed Meckel's diverticulum and abscess formation (surgery proven). Intravenous- and oral-enhanced CT scan shows a contrast-filled tubular diverticulum with a "mucosal triangular plateau" pattern at its ileal attachment (curved arrow); there is a fluid-filled collection at the diverticulum's tip and associated fat stranding (arrowhead).

fluid collection, with nonenhancing walls, in direct contact with the terminal ileum is highly suggestive of a necrotic Meckel's diverticulum (Fig. 5).

These particular CT patterns of an inflamed Meckel's diverticulum are sometimes difficult to differentiate from other acute digestive inflammations in the same region, such as appendicitis, ileal diverticulitis, or Crohn's disease.

The inflamed Meckel's diverticulum is always centered on a loop of terminal ileum, with locally surrounding mesenteric inflammatory changes. Other helpful signs in suggesting a Meckel's diverticulum abscess, rather than an abscess of appendicular origin, are the normal aspect of the caecum and appendix, delineated by oral contrast material (Fig. 6).

Localization of the abscess on the antimesenteric side of the ileum is very characteristic of an inflamed Meckel's diverticulum and helps with differentiation from ileal diverticulitis [6].

Ileal Crohn's disease with its complications (inflammation, perforation, obstruction) may present with clinical signs similar to complicated Meckel's diverticulum. CT scan of patients with Crohn's disease demonstrates extensive involvement of small bowel proximal and distal to the site of complication, whether it is an abscess or a perforation with the presence of extra-digestive air in the mesentery. Moreover, Crohn's disease will induce fibrofatty modification of the mesentery, which is not found in patients with an inflamed Meckel's diverticulum [22]. Rarely, Crohn's disease and Meckel diverticulum may coexist, with involvement of the diverticulum by terminal ileitis causing diverticulitis, which may progress towards abscess and perforation [23] (Fig. 7). 
Fig. 3a, b A 33-year-old woman with surgically proven perforated Meckel's diverticulum. a Intravenous- and oral-enhanced CT shows the perforated Meckel's diverticulum as a gascontaining collection in the right lower quadrant (asterisk), situated between the ileal loops and surrounded by fat stranding ( $a r-$ row). b Coronal reconstructed CT demonstrates the perforated diverticulum (asterisk), contiguous with a thickened ileal loop (arrow).
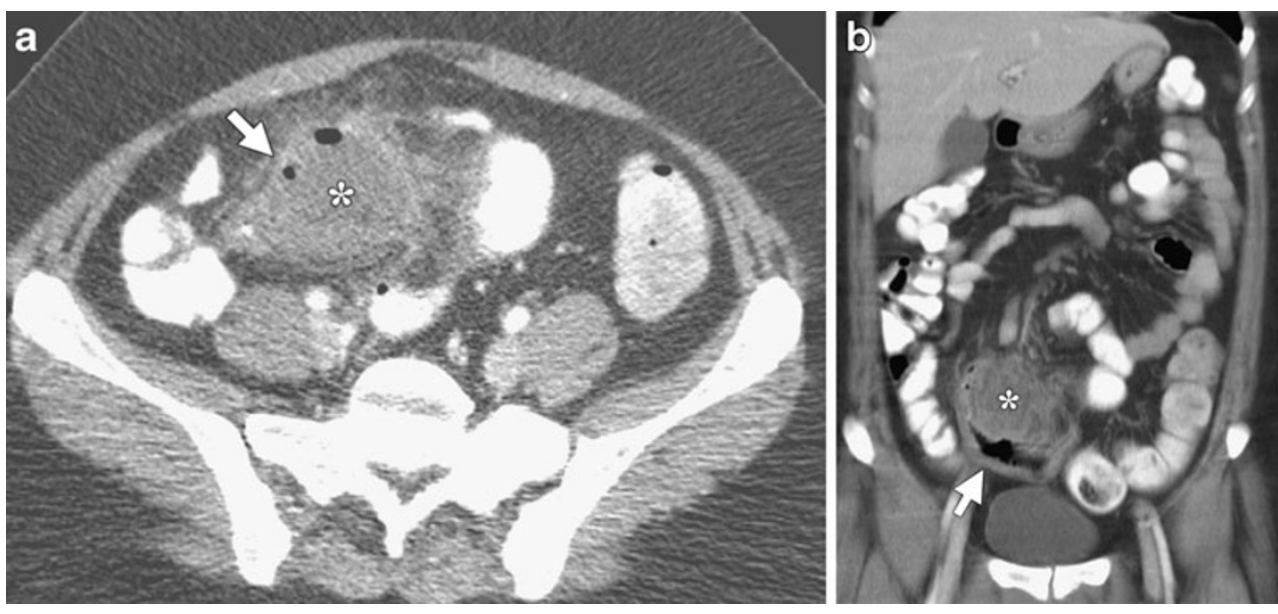

\section{Hemorrhage}

Bleeding is reported to be the most common complication of Meckel's diverticula in children and young adults. Hemorrhage results from ulceration of normal ileal mucosa adjacent to a diverticulum lined with acid-secreting gastric ectopic mucosa. Bleeding may be occult and intermittent, and patients usually present different degrees of anemia, or it may be brisk and important, leading to hemodynamic impairment.

In emergency setting, CT examination is a very useful tool in diagnosing both the localization and the source of overt gastrointestinal hemorrhage.

$\mathrm{CT}$ is fast, non-invasive, and requires no specific preparation of the patient. CT is ready available in the emergency department, and patients can be imaged during active clinical bleeding, thus increasing the chances of correctly diagnosing the origin of the hemorrhage [13].

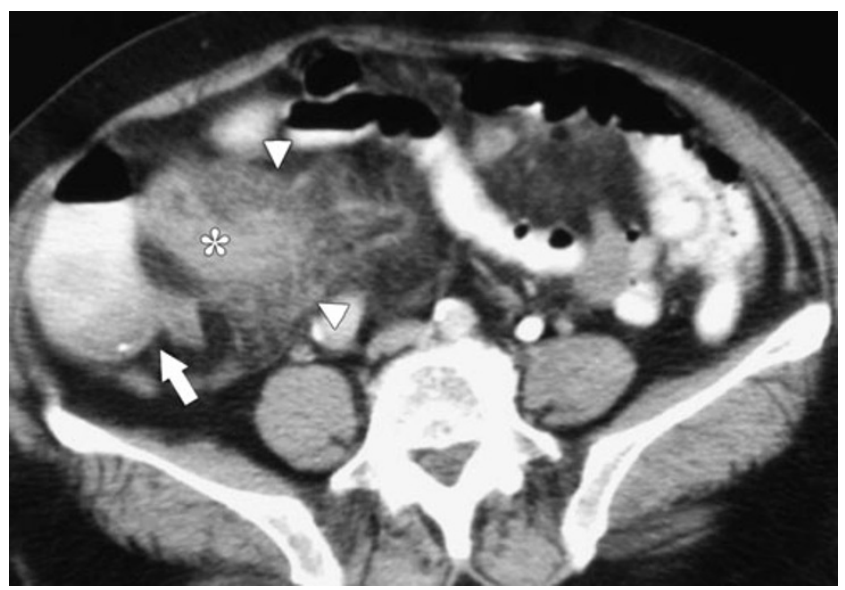

Fig. 4 A 71-year-old woman with surgically proven inflamed Meckel's diverticulum with tip perforation. Intravenous- and oralenhanced CT shows the diverticulum as a tubular, thickened-wall, blind-ending digestive structure (asterisk) surrounded by an important fat infiltration (arrowheads); the diverticulum is situated in the right lower quadrant. Caecum and terminal ileum are normal (arrow).
When active extravasation of contrast occurs in the terminal ileal loops, without underlying anomalies seen on $\mathrm{CT}$, the diagnosis of bleeding Meckel's diverticulum is most
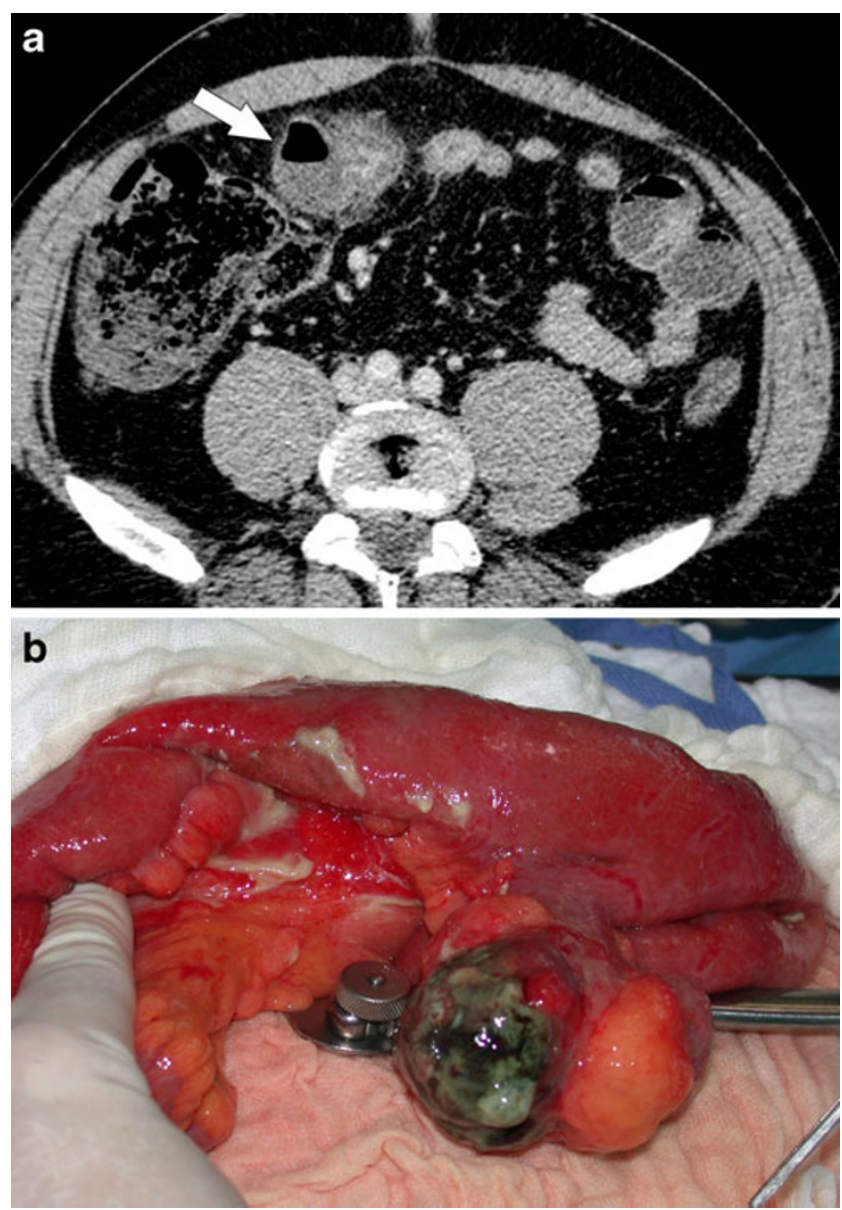

Fig. 5a, b A 44-year-old man with surgically proven necrotic Meckel's diverticulum (images courtesy of Dr. K. Bider and Dr. C. Beynon). a Intravenous-enhanced CT shows the inflamed diverticulum as an air-fluid collection (arrow) with thin, nonenhancing wall adjacent to an ileal loop. b Photograph taken during surgery before opening the specimen shows a $7 \mathrm{~cm}$ large, necrotic Meckel's diverticulum. 

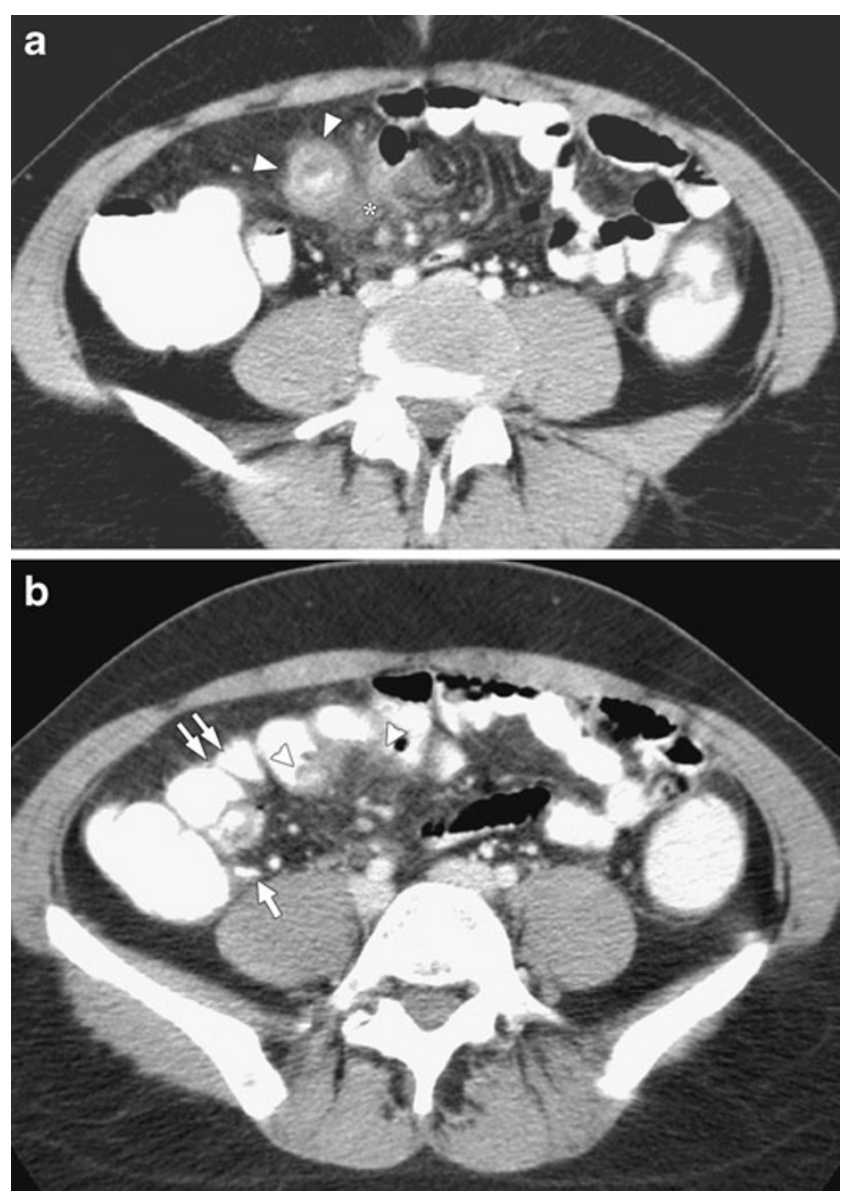

Fig. 6a, b A 32-year-old woman with surgically proven Meckel's diverticulitis. a Intravenous- and oral-enhanced CT shows the inflamed diverticulum as a round, contrast-filled, thickened-wall collection (arrowheads) in continuation with an ileal loop; associated fat stranding (asterisk) is also demonstrated. b CT scan at a higher level than a shows the peridiverticular inflammatory changes (arrowheads) along with normal aspect of the appendix (arrow) and of the ileal loops proximal to the inflamed diverticulum (double arrows).

likely [24]. The differential diagnosis of an active hemorrhage in the terminal ileum takes into consideration bleeding from ruptured aneurysm of the ileocolic artery [25] or bleeding from vasculitis or angiodysplasia; the differential diagnosis also includes hemorrhage associated with an underlying small bowel tumor [26]. Bleeding Meckel's diverticulum is suggested when extravasated contrast accumulates into an outpouching of the terminal ileum [16].

A definitive diagnosis of active bleeding into a Meckel's diverticulum is made when intravenous-enhanced CT shows extravasation of the contrast at the diverticular neck (Fig. 8), with delayed contrast accumulation into the saccular structure attached to the terminal ileum [16].

\section{Obstruction}

Meckel's diverticulum may lead to bowel obstruction by several mechanisms: mesodiverticular bands, volvulus from umbilical attachment, hernia, or intussusception. In addition, different complications may coexist. For example, Meckel's diverticulitis may lead to small bowel obstruction; in this situation, CT scan demonstrates associated signs of diverticular and mesenteric inflammation at the transition point of the dilated small bowel loops (Fig. 9).

Obstruction related to intussusception is a rare condition in adults, and an underlying pathology is found in $70-90 \%$ of cases [27]. A Meckel's diverticulum that is invaginated into the small bowel may occasionally be the cause of intussusception $[28,29]$. In these cases, CT scan examination shows an abnormal multi-layered, targetlike image, with a diameter larger than the normal bowel, consistent with the intussusception (Fig. 10); however, as previously reported [2], the direct visualization of the Meckel's diverticulum as the lead point is often difficult to assess, and the differential diagnosis includes intussusception produced by benign (lipoma) or malignant (metastasis, lymphoma) tumors [28].

\section{Retention of foreign body}

Surgeons have previously reported cases of perforation of Meckel's diverticulum by foreign bodies such as a chicken bone or a toothpick [30,31], but, as far as we know, there is no imaging documentation of this rare complication.

In our series, we document for the first time by CT scan examination the presence of a swallowed needle inside a Meckel's diverticulum (Fig. 11). In this case, the Meckel's diverticulum is recognizable as a long, tubular blind-ending structure, in continuation with an ileal loop. Presence of the needle at the collar of the diverticulum supports the

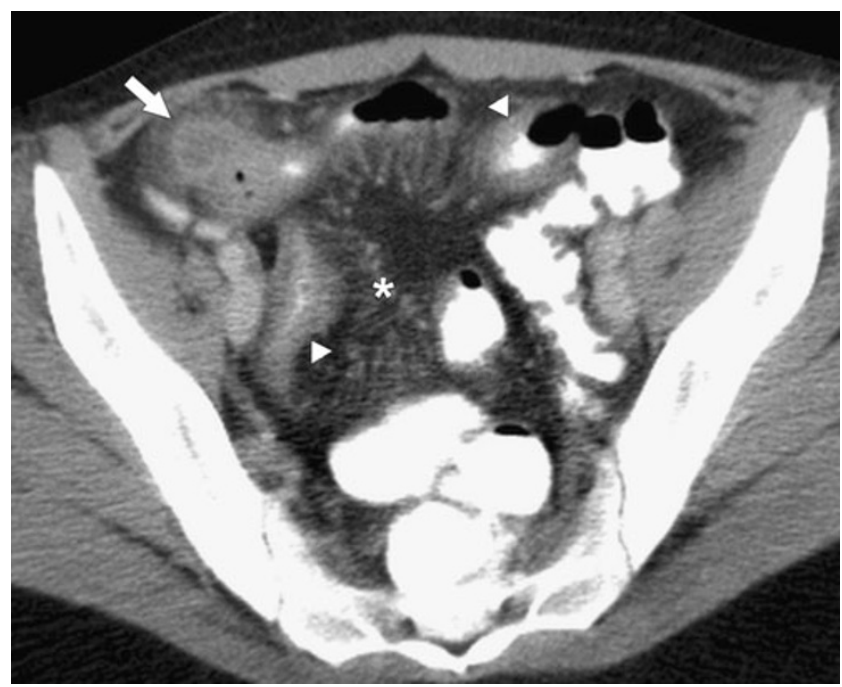

Fig. 7 A 22-year-old woman with surgically proven perforated Meckel's diverticulitis and Crohn's disease. Intravenous- and oralenhanced CT shows the collection due to the perforated Meckel's diverticulum (arrow) in the right lower quadrant. Thickened-wall ileal loops (arrowheads) and prominently dilated mesenteric vessels (asterisk), proving active Crohn's disease are also demonstrated. 

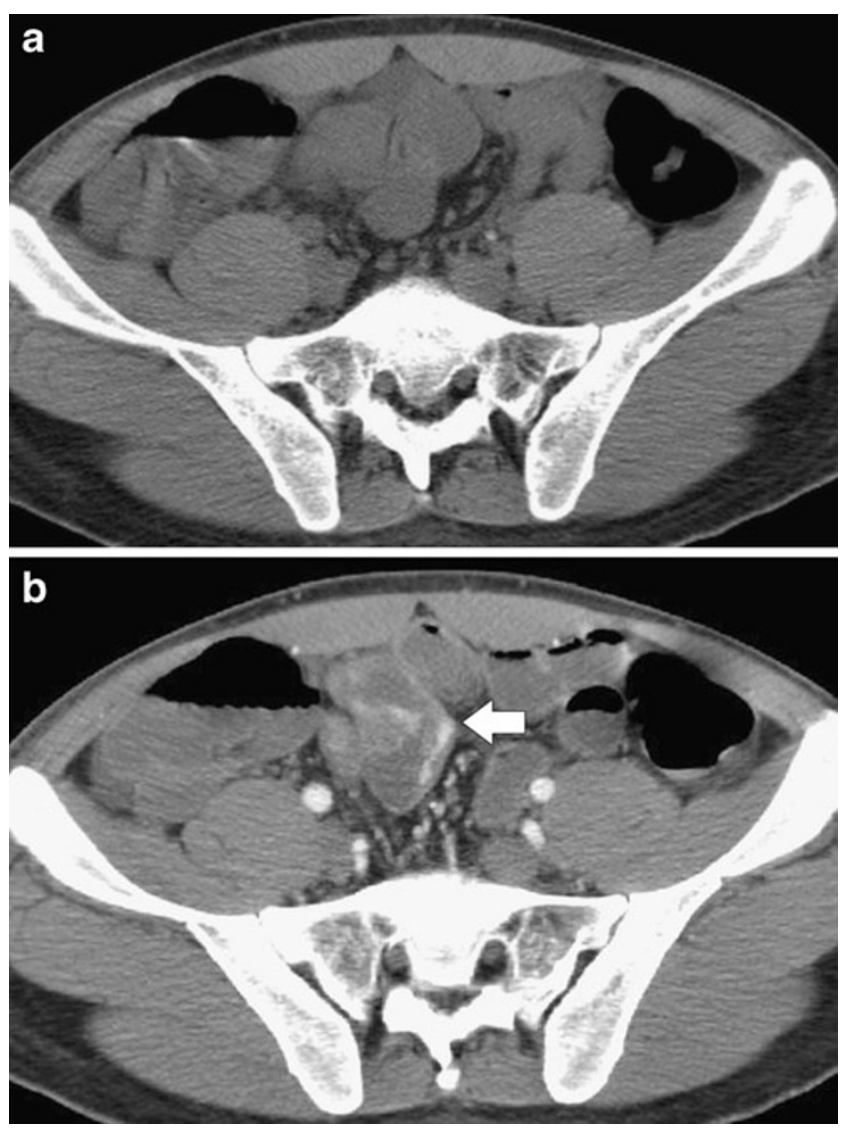

Fig. 8a, b A 22-year-old man with massive gastrointestinal bleeding due to heterotopic gastric mucosa in a Meckel's diverticulum (surgery proven). a Nonenhanced CT scan at the same level as b shows no spontaneously dense material in the bowel lumen. b Intravenousenhanced CT scan shows contrast extravasation seen as linear hyperattenuating pooling of contrast at the neck of the Meckel's diverticulum (arrow), arising from a distal ileal loop.

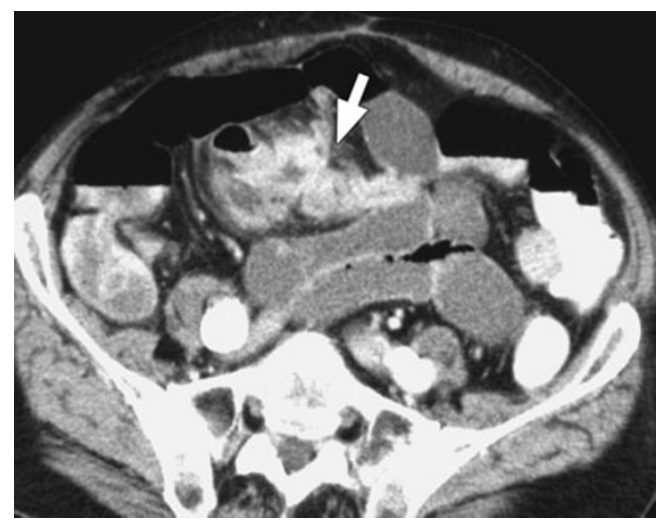

Fig. 9 A 63-year-old patient with small bowel obstruction due to inflammation of the Meckel's diverticulum (surgery proven). Intravenous abdominal CT shows the diverticulum as an enhancing, blindending digestive structure, with thick wall and fluid content (arrow); this finding was demonstrated to be an inflamed Meckel's diverticulum situated at the transition point with the dilated small bowel loops and surrounded by inflammatory changes in the mesenteric fat.
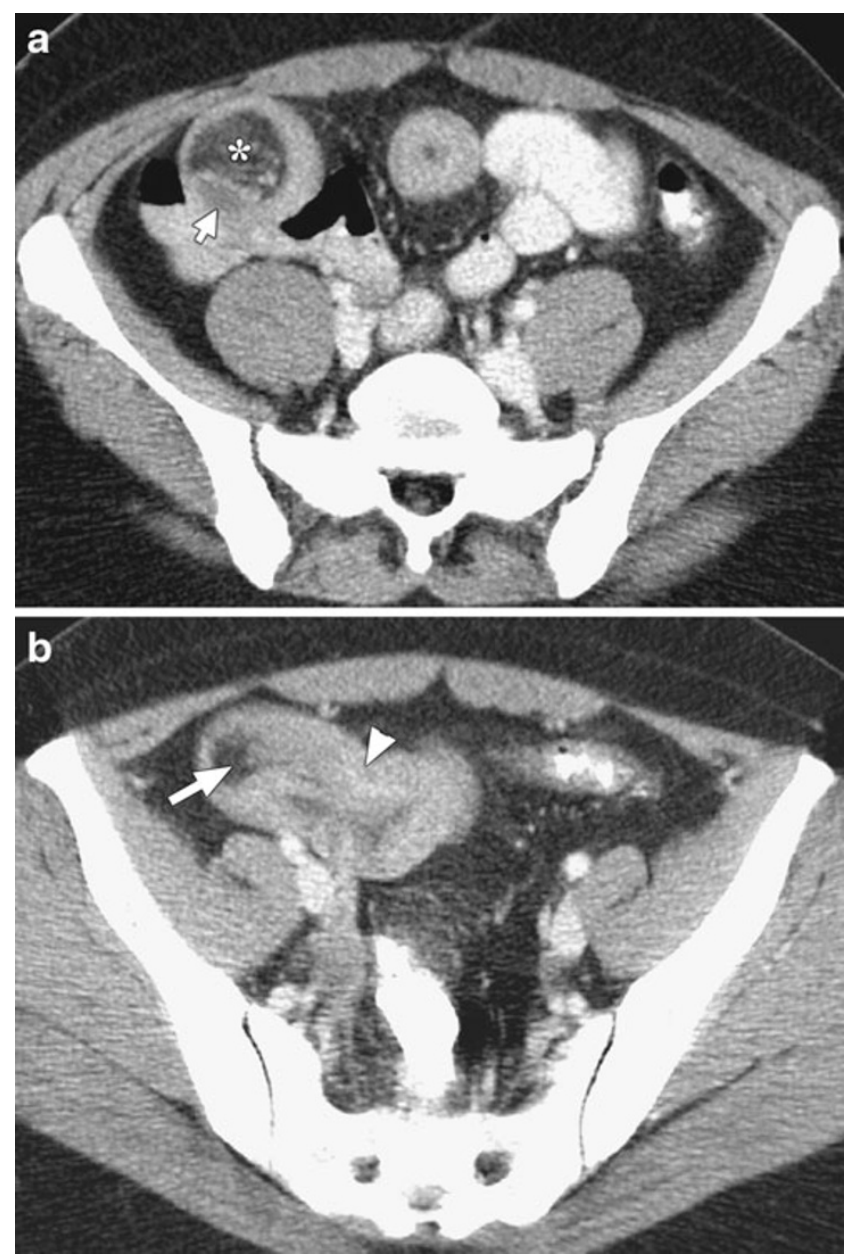

Fig. 10a, b A 39-year-old woman with surgically proven ileal intussusception related to the Meckel's diverticulum as the lead point. a Intravenous- and oral-enhanced CT scan shows the intussusception of the ileum (arrow) along with its mesentery (asterisk), creating the "target sign." b Image at a lower level than a demonstrates the invaginated mesenteric fat (arrow), along with thickening of the intussuscepted ileum (arrowhead).

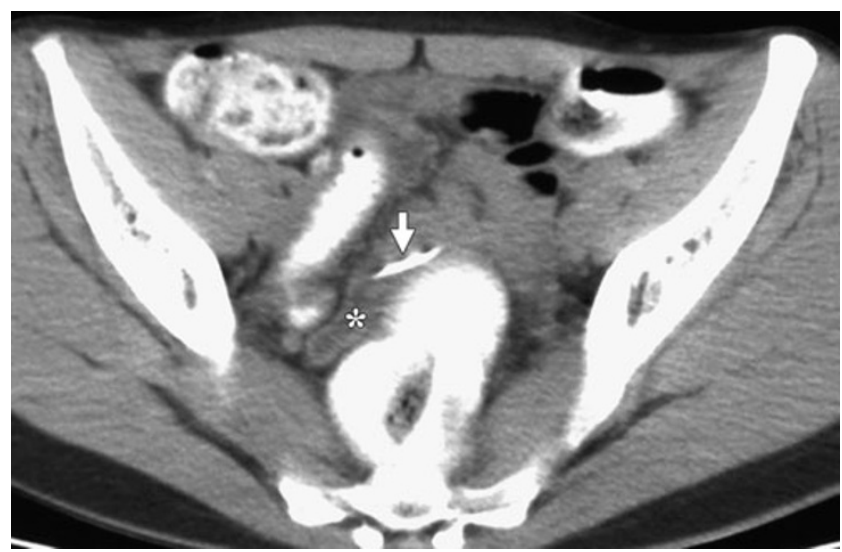

Fig. 11 A 20-year-old woman with surgically proven needle retention in a giant Meckel's diverticulum. Oral-enhanced CT shows the needle (arrow) located at the collar of a fluid-filled, blind-ended tubular structure (asterisk) that is the Meckel's diverticulum. 
hypothesis that the diverticular ileal attachment could act as a fixation point in the intestinal progression of different foreign bodies. Therefore, in these particular cases, the possible presence of a Meckel's diverticulum should be carefully sought between the distal ileal loops.

\section{Neoplasms}

Tumors arising in the Meckel's diverticulum are very rare; the reported histological types are carcinoid, gastrointestinal stromal tumors and adenocarcinomas [2]. We did not find any tumors developed in the Meckel's diverticulum in the patients included in our analysis.

\section{Conclusion}

Complicated Meckel's diverticula often constitute a challenging diagnosis for both the clinician and the radiologist. The presence of a Meckel's diverticulum is strongly suggested by $\mathrm{CT}$ scan demonstrating an abnormal outpouching, blind-ending digestive structure connected to the terminal ileum by a neck of varying caliber. Depending on the type of complication, the diverticulum may have surrounding mesenteric inflammatory changes or may look like a localized fluid or air-fluid collection, with normal aspect of the small bowel proximal and distal to the diverticular inflammation. The Meckel's diverticulum may be the source of active bleeding or may act as a lead point to intestinal obstruction and intussusception. CT findings of complicated Meckel's diverticulum are very polymorphic and should be remembered in the evaluation of adult patients with acute abdomen; we hope that our series will be helpful for the radiologist to better understand some of the presentations of this entity.

Acknowledgments The authors thank Dr. Katharina Bider and Dr. Christopher Beynon (Institute of Radiology, Triemli Hospital, Zürich) for their contribution to the illustration of this article.

\section{References}

1. Stone PA, Hofeldt MJ, Campbell JE, Vedula G, DeLuca JA, Flaherty SK (2004) Meckel diverticulum: ten-year experience in adults. South Med J 97:1038-1041

2. Levy AD, Hobbs CM (2004) Meckel diverticulum: radiologic features with pathologic correlation. Radiographics 24:565587

3. Cullen JJ, Kelly KA, Moir CR, Hodge DO, Zinsmeister AR, Melton LJ 3rd (1994) Surgical management of Meckel's diverticulum. An epidemiologic, population-based study. Ann Surg 220:564-568, discussion 568-569

4. Park JJ, Wolff BG, Tollefson MK, Walsh EE, Larson DR (2005) Meckel diverticulum: the Mayo Clinic experience with 1476 patients (1950-2002). Ann Surg 241:529-533
5. Rossi P, Gourtsoyiannis N, Bezzi M, Raptopoulos V, Massa R, Capanna G, Pedicini V, Coe M (1996) Meckel's diverticulum: imaging diagnosis. AJR Am J Roentgenol 166:567-573

6. Bennett GL, Birnbaum BA, Balthazar EJ (2004) CT of Meckel's diverticulitis in 11 patients AJR. Am J Roentgenol 182:625-629

7. Miele V, De Cicco ML, Andreoli C, Buffa V, Adami L, David V (2001) US and CT findings in complicated Meckel diverticulum. Radiol Med (Torino) 101:230-234

8. Feller AA, Movson J, Shah SA (2003) Meckel diverticulum: a geriatric disease masquerading as common gastrointestinal tract disorders. Arch Intern Med 163:2093-2096

9. Leschka S, Alkadhi H, Wildermuth S, Marincek B (2005) Multidetector computed tomography of acute abdomen. Eur Radiol 15:2435-2447

10. Olson DE, Kim YW, Donnelly LF (2009) CT findings in children with Meckel diverticulum. Pediatr Radiol 39:659-663

11. Mariani G, Pauwels EKJ, AlSharif A, Marchi S, Boni G, Barreca M, Bellini M, Grosso M, de Bortoli N, Mumolo G, Costa F, Rubello D, Strauss HW (2008) Radionuclide evaluation of the lower gastrointestinal tract. J Nucl Med 49:776-787

12. Graça BM, Freire PA, Brito JB, Ilharco JM, Carvalheiro VM, Caseiro-Alves F (2010) Gastroenterologic and radiologic approach to obscure gastrointestinal bleeding: how, why, and when? Radiographics 30:235-252

13. Laing CJ, Tobias T, Rosenblum DI, Banker WL, Tseng L, Tamarkin SW (2007) Acute gastrointestinal bleeding: emerging role of multidetector $\mathrm{CT}$ angiography and review of current imaging techniques. Radiographics 27:1055-1070

14. Mitchell AW, Spencer J, Allison DJ, Jackson JE (1998) Meckel's diverticulum: angiographic findings in 16 patients. AJR 170:1329-1333

15. Okazaki M, Higashihara H, Saida Y, Minami M, Yamasaki S, Sato S, Nagayama H (1993) Angiographic findings of Meckel's diverticulum: the characteristic appearance of the vitelline artery. Abdom Imaging 18:15-19

16. Danzer D, Gervaz P, Platon A, Poletti PA (2003) Bleeding Meckel's diverticulum diagnosis: an unusual indication for computed tomography. Abdom Imaging 28:631-633

17. Okazaki M, Higashihara H, Yamasaki S, Akita Y, Toriya H, Shirai Z (1990) Arterial embolization to control life-threatening hemorrhage from a Meckel's diverticulum. AJR 154:1257-1258

18. Elsayes KM, Menias CO, Harvin HJ, Francis IR (2007) Imaging manifestations of Meckel's diverticulum. AJR Am J Roentgenol 189:81-88

19. Yoon W, Jeong YY, Shin SS, Lim HS, Song SG, Jang NG, Kim JK, Kang HK (2006) Acute massive gastrointestinal bleeding: detection and localization with arterial phase multi-detector row helical CT. Radiology 239:160-167

20. Gayer G, Zissin R, Apter S, Shemesh E, Heldenberg E (1999) Acute diverticulitis of the small bowel: CT findings. Abdom Imaging 24:452-455

21. Hughes JA, Hatrick A, Rankin S (1998) Computed tomography findings in an inflamed Meckel diverticulum. Br J Radiol 71:882883

22. Furukawa A, Saotome T, Yamasaki M, Maeda K, Nitta N, Takahashi M, Tsujikawa T, Fujiyama Y, Murata K, Sakamoto T (2004) Cross-sectional imaging in Crohn disease. Radiographics 24:689-702

23. Schwenter F, Gervaz P, De Saussure P, MacKee T, Morel P (2009) Perforated Meckel's diverticulitis complicating active Crohn's ileitis: a case report. J Med Case Reports 3:12

24. Munroe CA, Copland A, Pai R, Friedland S, Triadafilopoulos G (2010) Meckel's diverticulum with gastrointestinal bleeding: role of computed tomography in diagnosis. Dig Dis Sci 55(2):242-244

25. Poletti PA, Vargas MI, Becker CD (2001) Successful treatment of a ruptured mycotic aneurysm of the ileocolic artery with trans- 
catheter embolization and antibiotic therapy. Abdom Imaging 26:651-653

26. Filippone A, Cianci R, Milano A, Valeriano S, Di Mizio V, Storto ML (2008) Obscure gastrointestinal bleeding and small bowel pathology: comparison between wireless capsule endoscopy and multidetector-row CT enteroclysis. Abdom Imaging 33:398-406

27. Azar T, Berger DL (1997) Adult intussusception. Ann Surg 226:134-138

28. Kim YH, Blake MA, Harisinghani MG, Archer-Arroyo K, Hahn PF, Pitman MB, Mueller PR (2006) Adult intestinal intussuscep- tion: CT appearances and identification of a causative lead point. Radiographics 26:733-744

29. Hamada T, Ishida O, Yasutomi M (1996) Inverted Meckel diverticulum with intussusception: demonstration by CT. J Comput Assist Tomogr 20:287-289

30. Yagci G, Cetiner S, Tufan T (2004) Perforation of Meckel's diverticulum by a chicken bone, a rare complication: report of a case. Surg Today 34:606-608

31. Zingg U, Vorburger S, Metzger U (2000) Perforation of Meckel's diverticulum by a toothpick. Chirurg 71:841-843 